\title{
Author Correction: Targeting wild-type KRAS-amplified gastroesophageal cancer through combined MEK and SHP2 inhibition
}

Gabrielle S. Wong, Jin Zhou, Jie Bin Liu, Zhong Wu, Xinsen Xu, Tianxia Li, David Xu, Steven E. Schumacher, Jens Puschhof, James McFarland, Charles Zou, Austin Dulak, Les Henderson, Peng Xu, Emily O'Day, Rachel Rendak, Wei-li Liao, Fabiola Cecchi, Todd Hembrough, Sarit Schwartz, Christopher Szeto, Anil K. Rustgi, Kwok-Kin Wong, J. Alan Diehl(10, Karin Jensen, Francesco Graziano, Annamaria Ruzzo, Shaunt Fereshetian, Philipp Mertins, Steven A. Carr (D), Rameen Beroukhim, Kenichi Nakamura, Eiji Oki, Masayuki Watanabe, Hideo Baba, Yu Imamura, Daniel Catenacci and Adam J. Bass

Correction to: Nature Medicine https://doi.org/10.1038/s41591-018-0022-x, published online 28 May 2018.

In the Supplementary Information originally published with this article, a lane was missing in the $\beta$-actin blot in Supplementary Fig. 2. The lane has been added. The error has been corrected in the Supplementary Information associated with this article.

\section{Author Correction: Metformin reverses established lung fibrosis in a bleomycin model}

Sunad Rangarajan (D), Nathaniel B. Bone, Anna A. Zmijewska, Shaoning Jiang (D), Dae Won Park, Karen Bernard, Morgan L. Locy (D), Saranya Ravi, Jessy Deshane, Roslyn B. Mannon (D, Edward Abraham, Victor Darley-Usmar, Victor J. Thannickal and Jaroslaw W. Zmijewski (D)

Correction to: Nature Medicine https://doi.org/10.1038/s41591-018-0087-6, published online 2 July 2018.

In the version of this article originally published, a grant was omitted from the Acknowledgements section. The following sentence should have been included: "R.B.M. was supported by a Department of Veterans Affairs Merit Award (5I01BX003272)." The error has been corrected in the HTML and PDF versions of this article.

\section{Author Correction: Mutations in the SWI/SNF complex induce a targetable dependence on oxidative phosphorylation in lung cancer}

Yonathan Lissanu Deribe, Yuting Sun, Christopher Terranova, Fatima Khan, Juan Martinez-Ledesma, Jason Gay, Guang Gao, Robert A. Mullinax, Tin Khor, Ningping Feng, Yu-Hsi Lin (D), Chia-Chin Wu (D), Claudia Reyes, Qian Peng, Frederick Robinson, Akira Inoue, Veena Kochat, Chang-Gong Liu, John M. Asara, Cesar Moran, Florian Muller (D), Jing Wang, Bingliang Fang, Vali Papadimitrakopoulou, Ignacio I. Wistuba, Kunal Rai, Joseph Marszalek and P. Andrew Futreal

Correction to: Nature Medicine https://doi.org/10.1038/s41591-018-0019-5, published online 8 July 2018.

In the version of this article originally published, information regarding several funding sources was omitted from the Acknowledgements section. The following sentences should have been included: "This work was supported by the generous philanthropic contributions to The University of Texas MD Anderson Lung Cancer Moon Shots Program, the UT Lung SPORE 5 P50 CA07090, and the MD Anderson Cancer Center Support Grant P30CA01667. V.P is supported by R01CA155196-01A1 from the National Cancer Institute." Also, reference 18 was incorrect. The original reference was: Kim, E. S. et al. The BATTLE trial: personalizing therapy for lung cancer. Cancer Discov. 1, 44-53 (2011). It should have been: Papadimitrakopoulou, V. et al. The BATTLE-2 study: a biomarker-integrated targeted therapy study in previously treated patients with advanced non-small-cell lung cancer. J Clin. Oncol. 34, 3638-3647 (2016). The errors have been corrected in the HTML and PDF versions of this article. 